\title{
Extending Partial Representations of Circle Graphs ${ }^{\star}$
}

\author{
Steven Chaplick ${ }^{1}$, Radoslav Fulek ${ }^{1}$, and Pavel Klavík ${ }^{2, \star \star}$ \\ 1 Department of Applied Mathematics, Faculty of Mathematics and Physics, \\ Charles University, Malostranské náměstí 25, 11800 Prague, Czech Republic \\ chaplick@kam.mff.cuni.cz, radoslav.fulek@gmail.com \\ 2 Computer Science Institute, Faculty of Mathematics and Physics, \\ Charles University, Malostranské náměstí 25, 11800 Prague, Czech Republic \\ klavik@iuuk.mff.cuni.cz
}

\begin{abstract}
The partial representation extension problem is a recently introduced generalization of the recognition problem. A circle graph is an intersection graph of chords of a circle. We study the partial representation extension problem for circle graphs, where the input consists of a graph $G$ and a partial representation $\mathcal{R}^{\prime}$ giving some pre-drawn chords that represent an induced subgraph of $G$. The question is whether one can extend $\mathcal{R}^{\prime}$ to a representation $\mathcal{R}$ of the entire $G$, i.e., whether one can draw the remaining chords into a partially pre-drawn representation.

Our main result is a polynomial-time algorithm for partial representation extension of circle graphs. To show this, we describe the structure of all representation a circle graph based on split decomposition. This can be of an independent interest.
\end{abstract}

\section{Introduction}

Graph drawings and visualizations are important topics of graph theory and computer science. A frequently studied type of representations are so-called intersection representations. An intersection representation of a graph represents its vertices by some objects and encodes its edges by intersections of these objects, i.e., two vertices are adjacent if and only if the corresponding objects intersect. Classes of intersection graphs are obtained by restricting these objects; e.g., interval graphs are intersection graphs of intervals of the real line, string graphs are intersection graphs of strings in plane, and so on. These representations are well-studied; see e.g. [25].

For a fixed class $\mathcal{C}$ of intersection-defined graphs, a very natural computational problem is recognition. It asks whether an input graph $G$ belongs to $\mathcal{C}$. In this paper, we study a recently introduced generalization of this problem called partial representation extension [19]. Its input gives with $G$ a part of the representation and the problem asks whether this partial representation can be extended to a representation of the entire $G$; see Fig. 1 for an illustration. We show that this problem can be solved in polynomial time for the class of circle graphs.

\footnotetext{
* Supported by ESF Eurogiga project GraDR as GAČR GIG/11/E023.

** Supported by Charles University as GAUK 196213.
}

S. Wismath and A. Wolff (Eds.): GD 2013, LNCS 8242, pp. 131-142, 2013.

(C) Springer International Publishing Switzerland 2013 

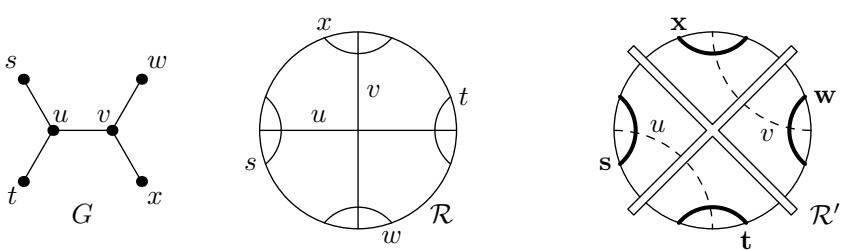

Fig. 1. On the left, a circle graph $G$ with a representation $\mathcal{R}$ is given. A partial representation $\mathcal{R}^{\prime}$ given on the right with the pre-drawn chords $\mathbf{s}, \mathbf{t}, \mathbf{w}$, and $\mathbf{x}$ is not extendible. The chords are depicted as arcs to make the figure more readable.

Circle Graphs. Circle graphs are intersection graphs of chords of a circle. They were first considered by Even and Itai 12 in the early 1970s in study of stack sorting techniques. Other motivations are due to their relations to Gauss words [1] (see Fig. 21) and matroid representations [10 5]. Circle graphs are also important regarding rank-width $[22$.

Let $\chi(G)$ denote chromatic number of $G$, and let $\omega(G)$ denote the clique-number of $G$. Trivially we have $\omega(G) \leq \chi(G)$ and the graphs for which every induced subgraph satisfies equality are the well-known perfect graphs [6]. In general, the difference between these two numbers can be arbitrarily high, e.g, there is a triangle-free graph with arbitrary high chromatic number. Circle graphs are known to be almost perfect which means that $\chi(G) \leq f(\omega(G))$ for some function $f$. The best known result for circle graphs [20] states that $f(k)$ is $\Omega(k \log k)$ and $\mathcal{O}\left(2^{k}\right)$.

The complexity of recognition of circle graphs was a long standing open problem; see [25] for an overview. The first results, e.g., [12], gave existential characterizations which did not give polynomial-time algorithms. The mystery whether circle graphs can be recognized in polynomial time frustrated mathematicians for some years. It was resolved in the mid-1980s and several polynomial-time algorithms were discovered [1321] (in time $\mathcal{O}\left(n^{7}\right)$ and similar). Later, a more efficient algorithm 24] based on split decomposition was given, and the current state-of-the-art recognition algorithm [14] runs in a quasi-linear time in the number of vertices and the number of edges of the graph.

The Partial Representation Extension Problem. It is quite surprising that this very natural generalization of the recognition problem was considered only recently. It is currently an active area of research which is inspiring a deeper investigation of many classical graph classes. For instance, a recent result of Angelini et al. 1] states that the problem is decidable in linear time for planar
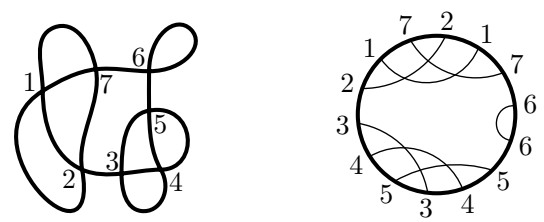

Fig. 2. A self-intersecting closed curve with $n$ intersections numbered $1, \ldots, n$ corresponds to a representation of circle graph with the vertices $1, \ldots, n$ where the endpoints of the chords are placed according to the order of the intersections along the curve. 
graphs. On the other hand, Fáry's Theorem claims that every planar graph has a straight-line embedding, but extension of such an embedding is NP-hard [23.

In the context of intersection-defined classes, this problem was first considered in [19] for interval graphs. Currently, the best known results are linear-time algorithms for interval graphs [3] and proper interval graphs [17, a quadratictime algorithm for unit interval graphs [17, and polynomial-time algorithms for permutation and function graphs 16. For chordal graphs (as subtree-in-a-tree graphs) several versions of the problems were considered [18] and all of them are NP-complete.

The Structure of Representations. To solve the recognition problem for $G$, one just needs to build a single representation. However, to solve the partial representation extension problem, the structure of all representations of $G$ must be well understood. A general approach used in the above papers is the following. We first derive necessary and sufficient constraints from the partial representation $\mathcal{R}^{\prime}$. Then we efficiently test whether some representation $\mathcal{R}$ satisfies these constraints. If none satisfies them, then $\mathcal{R}^{\prime}$ is not extendible. And if some $\mathcal{R}$ satisfies them, then it extends $\mathcal{R}^{\prime}$.

It is well-known that the split decomposition [8, Theorem 3] captures the structure of all representations of circle graphs. The standard recognition algorithms produce a special type of representations using split decomposition as follows. We find a split in $G$, construct two smaller graphs, build their representation recursively, and then join these two representations to produce $\mathcal{R}$. In Section 3, we give a simple recursive descriptions of all possible representations based on splits. Our result can be interpreted as "describing a structure like PQ-trees for circle graphs." It is possible that the proof techniques from other papers on circle graphs such as 7]14 would give a similar description. However, these techniques are more involved than our approach which turned out to be quite elementary and simple.

Restricted Representations. The partial representation extension problem belongs to a larger group of problems dealing with restricted representations of graphs. These problems ask whether there is some representation of an input graph $G$ satisfying some additional constraints. We describe two examples of these problems.

An input of the simultaneous representations problem, shortly SIM, consists of graphs $G_{1}, \ldots, G_{k}$ with some vertices common for all the graphs. The problem asks whether there exist representations $\mathcal{R}_{1}, \ldots, \mathcal{R}_{k}$ representing the common vertices the same. This problem is polynomially solvable for permutation and comparability graphs [15]. They additionally show that for chordal graphs it is NP-complete when $k$ is part of the input and polynomially solvable for $k=2$. For interval graphs, a linear-time algorithm is known for $k=2[3]$ and the complexity is open in general. For some classes, these problems are closely related to the partial representation extension problems. For example, there is an FPT algorithm for interval graphs with the number of common vertices as the parameter [19], and partial representations of interval graphs can be extended in linear time by reducing it to a corresponding simultaneous representations problem [3]. 
The bounded representation problem 17] prescribes bounds for each vertex of the input graph and asks whether there is some representation satisfying these bounds. For circle graphs, the input specifies a pair of $\operatorname{arcs}\left(A_{v}, A_{v}^{\prime}\right)$ of the circle for each chord $v$ and a solution is required to have one endpoint of $v$ in $A_{v}$ and the other one in $A_{v}^{\prime}$. This problem is clearly a generalization of partial representation extension since one can describe a partial representation using singleton arcs. It is known to be polynomially solvable for interval and proper interval graphs [2, and surprisingly it is NP-complete for unit interval graphs [17. The complexity for other classes of graphs is not known.

Our Results. We study the following problem (see Section 2 for definitions):

Problem: Partial Representation Extension - RepExt(CIRCLE)

Input: A circle graph $G$ and a partial representation $\mathcal{R}^{\prime}$.

Output: Is there a representation $\mathcal{R}$ of $G$ extending $\mathcal{R}^{\prime}$ ?

In Section 3, we describe a simple structure of all representations. This is used in Section 4 to obtain our main algorithmic result:

Theorem 1. The problem REPExT(CIRCLE) can be solved in polynomial time.

To spice up our results, we show in the full version of the paper the following.

Proposition 2. For $k$ part of the input, the problem $\operatorname{SIM}(\mathrm{CIRCLE})$ is NP-complete.

Corollary 3. The problem $\operatorname{SIM}(\mathrm{CIRCLE})$ is $\mathrm{FPT}$ in the size of the common subgraph.

\section{Definitions and Preliminaries}

Circle Representations. A circle graph representation $\mathcal{R}$ is a collection of chords a circle $\left\{C_{u} \mid u \in V(G)\right\}$ such that $C_{u}$ intersects $C_{v}$ if and only if $u v \in E(G)$. A graph is a circle graph if it has a circle representation, and we denote the class of circle graphs by CIRCLE.

Notice that the representation of a circle graph is completely determined by the circular order of the endpoints of the chords in the representation, and two chords $C_{u}$ and $C_{v}$ cross if and only if their endpoints alternate in this order. For convenience we label both endpoints of the chord representing a vertex by the same label as the vertex.

A partial representation $\mathcal{R}^{\prime}$ is a representation of an induced subgraph $G^{\prime}$. The vertices of $G^{\prime}$ are pre-drawn vertices and the chords of $\mathcal{R}^{\prime}$ are pre-drawn chords. A representation $\mathcal{R}$ extends $\mathcal{R}^{\prime}$ if $C_{u}=C_{u}^{\prime}$ for every $u \in V\left(G^{\prime}\right)$.

Word Representations. A sequence $\tau$ over an alphabet of symbols $\Sigma$ is a word. A circular word represents a set of words which are cyclical shifts of one another. In the sequel, we represent a circular word by a word from its corresponding set of words. We denote words and circular words by small Greek letters.

For a word $\tau$ and a symbol $u$ we write $u \in \tau$, if $u$ appears at least once in $\tau$. Thus, $\tau$ is also used to denote the set of symbols occurring in $\tau$. A word $\tau$ is a subword of $\sigma$, if $\tau$ appears consecutively in $\sigma$. A word $\tau$ is a subsequence of $\sigma$, 
if the word $\tau$ can be obtained from $\sigma$ by deleting some symbols. We say that $u$ alternates with $v$ in $\tau$, if $u v u v$ or $v u v u$ is a subsequence of $\tau$. The corresponding definitions also apply to circular words. If $\sigma$ and $\tau$ are two words, we denote their concatenation by $\sigma \tau$.

From now on each representation $\mathcal{R}$ of $G$ corresponds to the unique circular word $\tau$ over $V$. The word $\tau$ is obtained by the circular order of the endpoints of the chords in $\mathcal{R}$ as they appear along the circle when traversed clockwise. The occurrences of $u$ and $v$ alternate in $\tau$ if and only if $u v \in E(G)$. For example $\mathcal{R}$ in Fig. 1 corresponds to the circular word $\tau=$ susxvxtutwvw.

Let $G$ be a circle graph, and let $\mathcal{R}$ be its representation with the corresponding circular word $\tau$. If $G^{\prime}$ is an induced subgraph of $G$, then the subsequence of $\tau$ consisting of the vertices in $G^{\prime}$ is a circular word $\sigma$. This $\sigma$ corresponds to a representation $\mathcal{R}^{\prime}$ of $G^{\prime}$ which is extended by $\mathcal{R}$.

\section{Structure of Representations of Splits}

Let $G$ be a connected graph. A split of $G$ is a partition of the vertices of $G$ into four parts $A, B, \mathfrak{s}(A)$ and $\mathfrak{s}(B)$, such that:

- For every $a \in A$ and $b \in B$, we have $a b \in E(G)$.

- There is no edge between $\mathfrak{s}(A)$ and $B \cup \mathfrak{s}(B)$, and between $\mathfrak{s}(B)$ and $A \cup \mathfrak{s}(A)$.

- Both sides of the split have at least two vertices: $|A \cup \mathfrak{s}(A)| \geq 2$ and $\mid B \cup$ $\mathfrak{s}(B) \mid \geq 2$.

Fig. 3 shows two possible representations of a split. Notice that a split is uniquely determined just by the sets $A$ and $B$, since $\mathfrak{s}(A)$ consists of connected components of $G \backslash(A \cup B)$ attached to $A$, and similarly for $\mathfrak{s}(B)$ and $B$. We refer to this split as a split between $A$ and $B$.

In this section, we examine the recursive structure of every possible representation of $G$ based on splits.

\subsection{Split Structure of a Representation}

Let $\mathcal{R}$ be a representation of a graph $G$ with a split between $A$ and $B$. The representation $\mathcal{R}$ corresponds to a unique circular word $\tau$ and we consider the
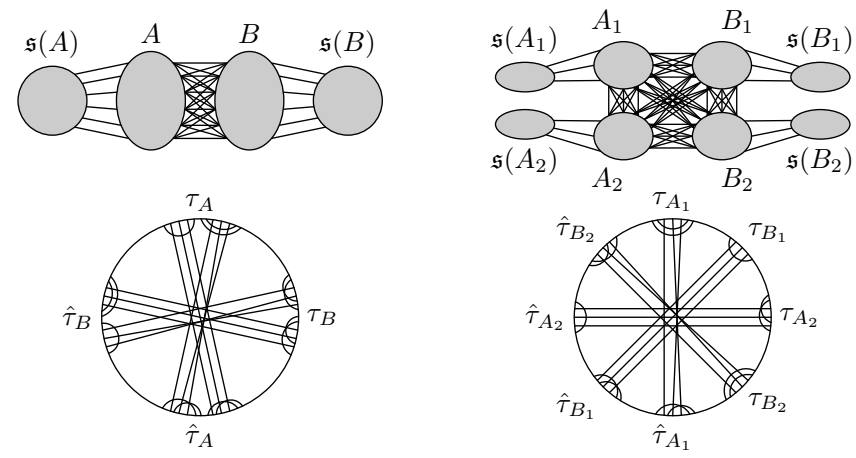

Fig. 3. Two different representations of $G$ with the split between $A$ and $B$. 
circular subsequence $\gamma$ induced by $A \cup B$. The maximal subwords of $\gamma$ consisting of $A$ alternate with the maximal subwords of $\gamma$ consisting of $B$. We denote all these maximal subwords $\gamma_{1}, \ldots, \gamma_{2 k}$ according to their circular order; so $\gamma=$ $\gamma_{1} \gamma_{2} \cdots \gamma_{2 k}$. Without loss of generality, we assume that $\gamma_{1}$ consists of symbols from $A$. We call $\gamma_{i}$ an $A$-word when $i$ is odd, and a $B$-word when $i$ is even.

We first investigate for each $\gamma_{i}$ which symbols it contains.

Lemma 4. For the subwords $\gamma_{1}, \ldots, \gamma_{k}$ the following holds:

(a) Each $\gamma_{i}$ contains each symbol at most once.

(b) The opposite words $\gamma_{i}$ and $\gamma_{i+k}$ contains the same symbols.

(c) Let $i \neq j$. If $x \in \gamma_{i}$ and $y \in \gamma_{j}$, then $x y \in E(G)$.

Proof (Sketch). These three properties are easily forced by the structure of the split; see the full version.

If $A, B \subset V(G)$ give rise to a split in $G$, we call the vertices of $A$ and $B$ the long vertices with respect to the split between $A$ and $B$. Similarly the vertices $\mathfrak{s}(A)$ and $\mathfrak{s}(B)$ are called short vertices with respect to the split between $A$ and $B$. In the sequel, if the split is clear from the context, we will just call some vertices long and some vertices short.

Consider a connected component $C$ of $\mathfrak{s}(A)$ (for a component of $\mathfrak{s}(B)$ the same argument applies) and consider the subsequence of $\tau$ induced by $A \cup B \cup$ $C$. By Lemma 4(a)-(b) and the fact that no vertex of $\mathfrak{s}(A)$ is adjacent to $B$, this subsequence almost equals $\gamma$. The only difference is that one subword $\gamma_{i}$ is replaced by a subword which additionally contains all occurrences of the vertices of $C$. By accordingly adding the vertices of all components of $\mathfrak{s}(A)$ and $\mathfrak{s}(B)$ to $\gamma$, we get $\tau$. Thus, $\tau$ consists of the circular subwords $\tau_{1}, \ldots, \tau_{2 k}$ concatenated in this order, where $\tau_{i}$ is obtained from $\gamma_{i}$ by adding the components of $\mathfrak{s}(A)$ or $\mathfrak{s}(B)$ attached to it. In particular, we also have the following:

Lemma 5. If two long vertices $x, y \in A$ are connected by a path having the internal vertices in $\mathfrak{s}(A)$, then $x$ and $y$ belong to the same pair $\gamma_{i}$ and $\gamma_{i+k}$ in any representation.

Proof. If $x$ and $y$ belong to different subwords $\gamma_{i}$ and $\gamma_{j}$, where $i<j$ and $j \neq i, i+k$, of $\gamma$, by Lemma 4(a)-(b) any path connecting $x$ and $y$ has an internal vertex adjacent to a vertex of $B$. However, no vertex in $\mathfrak{s}(A)$ is adjacent to a vertex of $B$.

\subsection{Conditions Forced by a Split}

Now, we want to investigate the opposite relation. Namely, what can one say about a representation from the structure of a split? Suppose that $x$ and $y$ are two long vertices. We want to know the properties of $x$ and $y$ which force every representation $\mathcal{R}$ to have a subword $\gamma_{i}$ of $\gamma$ containing both $x$ and $y$.

We define a relation $\sim$ on $A \cup B$ where $x \sim y$ means that $x, y$ has to be placed in the same subword $\gamma_{i}$ of $\gamma$. This relation is given by two conditions:

(C1) Lemma 4(c) states that if $x y \notin E(G)$, then $x \sim y$, i.e., if $x$ and $y$ are placed in different subwords, then $C_{x}$ intersects $C_{y}$. 
(C2) Lemma 5 gives $x \sim y$ when $x$ and $y$ are connected by a non-trivial path with all the inner vertices in $\mathfrak{s}(A) \cup \mathfrak{s}(B)$.

Let us take the transitive closure of $\sim$, which we denote by $\sim$ thereby slightly abusing the notation. Thus, we obtain an equivalence relation $\sim$ on $A \cup B$. Notice that every equivalence class of $\sim$ is either fully contained in $A$ or in $B$. For the graph in Fig. 3 the relation $\sim$ has four equivalence classes $A_{1}, A_{2}, B_{1}$ and $B_{2}$.

Now, let $\Phi$ be an equivalence class of $\sim$. We denote by $\mathfrak{s}(\Phi)$ the set consisting of all the vertices in the connected components of $G \backslash(A \cup B)$ which have a vertex adjacent to a vertex of $\Phi$. Since $\sim$ satisfies $(\mathrm{C} 2)$, we know that the sets $\mathfrak{s}(\Phi)$ of the equivalent classes of $\sim$ define a partition of $\mathfrak{s}(A) \cup \mathfrak{s}(B)$.

Recognition Algorithms Based on Splits. The splits are used in the current state-of-the-art algorithms for recognizing circle graphs. If a circle graph contains no split, it is called a prime graph. The representation of a prime graph is uniquely determined (up to the orientation of the circle) and can be constructed efficiently. There is an algorithm which finds a split between two sets $A$ and $B$ in linear time 9 . In fact, the entire split decomposition tree (i.e., the recursive decomposition tree obtained via splits) can be found in linear time. Usually the representation $\mathcal{R}$ is constructed as follows.

We define two graphs $G_{A}$ and $G_{B}$ where $G_{A}$ is a subgraph of $G$ induced by the vertices corresponding to $A \cup \mathfrak{s}(A) \cup\left\{v_{A}\right\}$ where the vertex $v_{A}$ is adjacent to all the vertices in $A$ and non-adjacent to all the vertices in $\mathfrak{s}(A)$, and $G_{B}$ is defined similarly for $B, \mathfrak{s}(B)$, and $v_{B}$. Then we apply the algorithm recursively on $G_{A}$ and $G_{B}$ and construct their representations $\mathcal{R}_{A}$ and $\mathcal{R}_{B}$; see Fig. 4. It remains to join the representations $\mathcal{R}_{A}$ and $\mathcal{R}_{B}$ in order to construct $\mathcal{R}$.

To this end we take $\mathcal{R}_{A}$ and replace $C_{v_{A}}$ by the representation of $B \cup \mathfrak{s}(B)$ in $\mathcal{R}_{B}$. More precisely, let the circular ordering of the endpoints of chords defined by $\mathcal{R}_{A}$ be $v_{A} \tau_{A} v_{A} \hat{\tau}_{A}$ and let the circular ordering defined by $\mathcal{R}_{B}$ be $v_{B} \tau_{B} v_{B} \hat{\tau}_{B}$. The constructed $\mathcal{R}$ has the corresponding circular ordering $\tau_{A} \tau_{B} \hat{\tau}_{A} \hat{\tau}_{B}$. It is easy to see that $\mathcal{R}$ is a correct circle representation of $G$.

Structure of All Representations. The above algorithm constructs a very specific representation $\mathcal{R}$ of $G$, and a representation like the one in Fig. 3 on the right cannot be constructed by the algorithm. In what follows we describe a structure of all the representations of $G$, based on different circular orderings of the classes of $\sim$. First, we show that every representation obtained in this way is a correct representation of $G$. Second, we prove that every representation $\mathcal{R}$ of $G$ can be constructed like this.
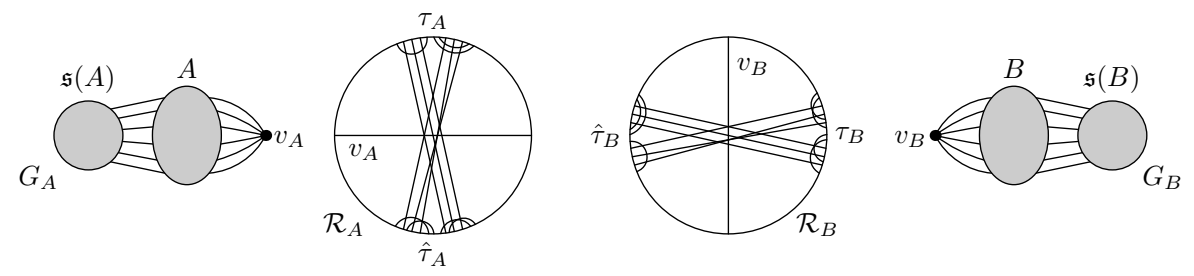

Fig. 4. The graphs $G_{A}$ and $G_{B}$ together with some constructed representations $\mathcal{R}_{A}$ and $\mathcal{R}_{B}$. By joining these representations, we get the left representation of Fig. 3 
We choose an arbitrary circular ordering $\Phi_{1}, \ldots, \Phi_{\ell}$ of the classes of $\sim$. Let $G_{i}$ be a graph constructed from $G$ by contracting the vertices of $V(G) \backslash\left(\Phi_{i} \cup\right.$ $\left.\mathfrak{s}\left(\Phi_{i}\right)\right)$ into one vertex $v_{i}$; i.e., $G_{i}$ is defined similarly to $G_{A}$ and $G_{B}$ above. Let $\mathcal{R}_{1}, \ldots, \mathcal{R}_{\ell}$ be arbitrary representations of $G_{1}, \ldots, G_{\ell}$. We join these representations as follows. Let $v_{i} \tau_{i} v_{i} \hat{\tau}_{i}$ be the circular ordering of $\mathcal{R}_{i}$. We construct $\mathcal{R}$ as the circular ordering

$$
\tau_{1} \tau_{2} \ldots \tau_{k-1} \tau_{k} \hat{\tau}_{1} \hat{\tau}_{2} \ldots \hat{\tau}_{k-1} \hat{\tau}_{k}
$$

In Fig. 3, we obtain the representation on the left by the circular ordering $A_{1} A_{2} B_{1} B_{2}$ of the classes of $\sim$ and the representation on the right by $A_{1} B_{1} A_{2} B_{2}$.

Lemma 6. Every circular ordering (11) constructed as above defines a circle representation of $G$.

Proof. Every long vertex $u \in \Phi_{i}$ alternates with $v_{i}$ in $\mathcal{R}_{i}$ and every short vertex $v \in \mathfrak{s}\left(\Phi_{i}\right)$ has both occurrences either in $\tau_{i}$, or in $\hat{\tau}_{i}$, since it is not adjacent to $v_{i}$. Thus, we get a correct representation $\mathcal{R}$ of $G$.

We now show that this approach can construct every representation of $G$.

Lemma 7. Let $\tau$ be the circular word corresponding to a representation $\mathcal{R}$ of $G$. Then the symbols of $\Phi_{i} \cup \mathfrak{s}\left(\Phi_{i}\right)$ form exactly two subwords of $\tau$.

Proof (Sketch). The conditions (C1) and (C2) imply consecutivity for some pairs $x$ and $y$. The statement is then proved by applying induction on the classes of $\sim$; see the full version for details.

Now, we are ready to prove the main structural proposition, which is inspired by Section IV.4 of the thesis of Naji [21].

Proposition 8. Let $\sim$ be the equivalence relation defined by (C1) and (C2) on $A \cup B$. Then every representation $\mathcal{R}$ corresponds to some circular ordering $\Phi_{1}, \ldots, \Phi_{\ell}$ and to some representations $\mathcal{R}_{1}, \ldots, \mathcal{R}_{\ell}$ of $G_{1}, \ldots, G_{\ell}$. More precisely, $\mathcal{R}$ can be constructed by arranging $\mathcal{R}_{1}, \ldots, \mathcal{R}_{\ell}$ as in (11): $\tau_{1} \ldots \tau_{k} \hat{\tau}_{1} \ldots \hat{\tau}_{k}$.

Proof. Let $\mathcal{R}$ be any representation with the corresponding circular word $\tau$. According to Lemma 7. we know $\Phi_{i} \cup \mathfrak{s}\left(\Phi_{i}\right)$ forms two subwords $\tau_{i}$ and $\hat{\tau}_{i}$ of $\tau$. For $i \neq j$, the edges between $\Phi_{i}$ and $\Phi_{j}$ form a complete bipartite graph. The subwords $\tau_{i}, \hat{\tau}_{i}, \tau_{j}$ and $\hat{\tau}_{j}$ alternate, i.e., appear as $\tau_{i} \tau_{j} \hat{\tau}_{i} \hat{\tau}_{j}$ or $\tau_{j} \tau_{i} \hat{\tau}_{j} \hat{\tau}_{i}$ in $\tau$. Thus, if we start from some point along the circle, the order of $\tau_{i}$ 's gives a circular ordering $\Phi_{1}, \ldots, \Phi_{\ell}$ of the classes. The representation $\mathcal{R}_{i}$ is given by the circular ordering $v_{i} \tau_{i} v_{i} \hat{\tau}_{i}$.

\section{Algorithm}

In this section, we give a polynomial-time algorithm for the partial representation extension problem of circle graphs. Our algorithm is based on the structure of all representations described in Section 3. We assume that the graph is connected and we deal with disconnected graphs in the full version of this paper.

Overview. Let $\tau^{\prime}$ be the circular word corresponding to the given partial rep. $\mathcal{R}^{\prime}$. We want to extend $\tau^{\prime}$ to a circular word $\tau$ corresponding to a rep. $\mathcal{R}$ of $G$. 
Our algorithm proceeds recursively via split decomposition.

1. If $G$ is prime, we have two possible representations (one is reversal of the other) and we test whether one of them is compatible with $\mathcal{R}^{\prime}$.

2. Otherwise, we find a split and compute the $\sim$ relation.

3. We test whether some ordering $\Phi_{1}, \ldots, \Phi_{\ell}$ of these classes along the circle is compatible with the partial representation $\mathcal{R}^{\prime}$. This order is partially prescribed by short chords and long chords of $\mathcal{R}^{\prime}$.

4. If no ordering is compatible, we stop and output "no". If there is an ordering which is compatible with $\mathcal{R}^{\prime}$, we recurse on the graphs $G_{1}, \ldots, G_{\ell}$ constructed according to the equivalence classes of $\sim$.

Now we describe everything in detail.

Splits. Now we assume that the graph is not prime, otherwise the problem is easy to solve (details will be given in the full version). A split between $A$ and $B$ is called trivial if for one side, let us say $A$, we have $|A|=1$ and $|\mathfrak{s}(A)|=1$. If $G$ contains only trivial splits, then we call it trivial. For technical purposes, we assume that the split is non-trivial, again the full version of this paper contains the details.

So we have a non-trivial split between $A$ and $B$ which can be constructed in polynomial time 9. We compute the equivalence relation $\sim$ and we want to find an ordering of its equivalence classes. For a class $\Phi$ of $\sim$, we define the extended class $\Psi$ of $\sim$ as $\Phi \cup \mathfrak{s}(\Phi)$. We can assume that each extended class has a vertex pre-drawn in the partial representation, otherwise any representation of it is good. So $\sim$ has $\ell$ equivalence classes, and all of them appear in $\tau^{\prime}$.

Now, $\tau^{\prime}$ is composed of $k$ maximal subwords, each containing only symbols of one extended class $\Psi$. We denote these maximal subwords as $\tau_{1}^{\prime}, \ldots, \tau_{k}^{\prime}$ according to their circular order in $\tau^{\prime}$, so $\tau^{\prime}=\tau_{1}^{\prime} \cdots \tau_{k}^{\prime}$. According to Proposition 8, each extended class $\Psi$ corresponds to at most two different maximal subwords. Also, if two extended classes $\Psi$ and $\hat{\Psi}$ correspond to two different maximal subwords, then occurrences of these subwords in $\tau^{\prime}$ alternate. Otherwise we reject the input.

\section{Case 1: An extended class corresponds to two maximal subwords.}

We denote this class by $\Psi_{1}$ and put this class as first in the ordering. By renumbering, we may assume that $\Psi_{1}$ corresponds to $\tau_{1}^{\prime}$ and $\tau_{t}^{\prime}$. Then one circular order of the classes can be determined as follows. We have $\Psi_{1}<\Psi$ for any other class $\Psi$. Let $\Psi_{i}$ and $\Psi_{j}$ be two distinct classes. If $\Psi_{i}$ corresponds to $\tau_{a}^{\prime}$ and $\Psi_{j}$ corresponds to $\tau_{b}^{\prime}$ such that either $a<b<t$ or $t<a<b$, we put $\Psi_{i}<\Psi_{j}$. We obtain the ordering of the classes as any linear extension of $<$. One can observe that $<$ is acyclic, otherwise the maximal subwords would not alternate correctly.

Now, we have ordered the extended classes $\Psi_{1}, \ldots, \Psi_{\ell}$ and the corresponding classes $\Phi_{1}, \ldots, \Phi_{\ell}$. We construct each $G_{i}$ with the vertices $\Psi_{i} \cup\left\{v_{i}\right\}$ as in Section 3.2, so $v_{i}$ is adjacent to $\Phi_{i}$ and non-adjacent to $\mathfrak{s}\left(\Phi_{i}\right)$. As the partial representation $\mathcal{R}_{i}^{\prime}$ of $G_{i}$, we put the word $v_{i} \tau_{i}^{\prime} v_{i} \tau_{j}^{\prime}$ where $\Psi_{i}$ corresponds to $\tau_{i}$ and $\tau_{j}$ (possibly one of them is empty). We test recursively, whether each representation $\mathcal{R}_{i}^{\prime}$ of $G_{i}$ is extendible to a representation of $\mathcal{R}_{i}$. If yes, we join $\mathcal{R}_{1}, \ldots, \mathcal{R}_{\ell}$ as in Proposition 8, Otherwise, the algorithm outputs "no". 
Lemma 9. For Case 1, the representation $\mathcal{R}^{\prime}$ is extendible if and only if the representations $\mathcal{R}_{1}^{\prime}, \ldots, \mathcal{R}_{\ell}^{\prime}$ of the graphs $G_{1}, \ldots, G_{\ell}$ are extendible.

Proof. Suppose that $\mathcal{R}$ extends $\mathcal{R}^{\prime}$. According to Proposition 8 , the representations of $\Psi_{1}, \ldots, \Psi_{\ell}$ are somehow ordered along the circle, and so we obtain representations $\mathcal{R}_{1}, \ldots, \mathcal{R}_{\ell}$ extending $\mathcal{R}_{1}^{\prime}, \ldots, \mathcal{R}_{\ell}^{\prime}$.

For the other implications, we just take $\mathcal{R}_{1}, \ldots, \mathcal{R}_{\ell}$ and put them in $\mathcal{R}$ together as in (11). The ordering $<$ was constructed exactly in such a way that $\mathcal{R}$ extends $\mathcal{R}^{\prime}$.

\section{Case 2: No extended class corresponds to two maximal subwords}

In this case, we have the ordering of the classes according to their appearance in $\tau^{\prime}$, so $\Psi_{i}$ corresponds to the subword $\tau_{i}^{\prime}$. According to Proposition 8 , we know that in any representation $\mathcal{R}$ of $G$ the class $\Psi_{i}$ corresponds to two subwords $\tau_{i}$ and $\hat{\tau}_{i}$. The difficulty here arises from the potential for $\tau_{i}^{\prime}$ to be a subsequence of only one of $\tau_{i}$ and $\hat{\tau}_{i}$.

We solve this as follows. Instead of constructing just one graph $G_{i}$ with one partial representation $\mathcal{R}_{i}^{\prime}$, we construct an additional graph $\widetilde{G}_{i}$ with a partial representation $\widetilde{\mathcal{R}}_{i}^{\prime}$ as follows. The graph $\widetilde{G}_{i}$ is $G_{i}$ with an additional leaf $w_{i}$ attached to $v_{i}$. The partial representation $\mathcal{R}_{i}^{\prime}$ corresponds to the word $\tau_{i}^{\prime} v_{i} v_{i}$ and the partial representation $\widetilde{\mathcal{R}}_{i}^{\prime}$ corresponds to $\tau_{i}^{\prime} w_{i} w_{i}$. The difference is that $\widetilde{\mathcal{R}}_{i}^{\prime}$ is less restrictive and only one endpoint of $v_{i}$ is prescribed (i.e., the location of the "other" end of $v_{i}$ is not restricted). We can easily observe that if $\mathcal{R}_{i}^{\prime}$ is extendible, then $\widetilde{\mathcal{R}}_{i}^{\prime}$ is also extendible.

The following lemma is fundamental for the algorithm, and it states that at most one class can be forced to use $\widetilde{G}_{i}$ with $\widetilde{\mathcal{R}}_{i}^{\prime}$, if $\tau^{\prime}$ is extendible:

Lemma 10. The representation $\mathcal{R}^{\prime}$ is extendible if and only if $\widetilde{\mathcal{R}}_{i}^{\prime}$ is extendible for some $i$ and $\mathcal{R}_{j}^{\prime}$ is extendible for all $j \neq i$.

Proof. Suppose that $\mathcal{R}_{j}$ corresponding to a word $v_{j} \tau_{j} v_{j} \hat{\tau}_{j}$ is an extension of $\mathcal{R}_{j}^{\prime}$ for $j \neq i$. And let $\mathcal{R}_{i}$ corresponding to a word $w_{i} v_{i} w_{i} \tau_{i} v_{i} \hat{\tau}_{i}$ be an extension of $\widetilde{\mathcal{R}}_{i}^{\prime}$. Then the representation $\mathcal{R}$ (after removing $w_{i}$ ) constructed as in (11) extends $\mathcal{R}^{\prime}$.

For the other implication, suppose that $\mathcal{R}$ extends $\mathcal{R}^{\prime}$. For contradiction, suppose that two distinct partial representations $\mathcal{R}_{i}^{\prime}$ and $\mathcal{R}_{j}^{\prime}$ are not extendible. According to Proposition 8 , the representation $\mathcal{R}$ gives a representation $\mathcal{R}_{i}$ corresponding to $v_{i} \tau_{i} v_{i} \hat{\tau}_{i}$ of $G_{i}$ and $\mathcal{R}_{j}$ corresponding to $v_{j} \tau_{j} v_{j} \hat{\tau}_{j}$ of $G_{j}$. But since both $\Psi_{i}$ and $\Psi_{j}$ correspond to single maximal words of $\tau^{\prime}$, we have that $\tau_{i}^{\prime}$ is a subsequence of $\tau_{i}$ or $\hat{\tau}_{i}$, or $\tau_{j}^{\prime}$ is a subsequence of $\tau_{j}$ or $\hat{\tau}_{j}$, and so $\mathcal{R}_{i}^{\prime}$ or $\mathcal{R}_{j}^{\prime}$ is extendible. Contradiction.

For the algorithm, we can efficiently test which of $\mathcal{R}_{i}^{\prime}$ and $\widetilde{\mathcal{R}}_{i}^{\prime}$ are extendible with the pseudocode of Algorithm 1 .

Analysis of the Algorithm. By using the established results, we show that the partial representation extension problem of circle graphs can be solved in polynomial time.

Lemma 11. The described algorithm correctly decides whether the partial representation $\mathcal{R}^{\prime}$ of $G^{\prime}$ is extendible. 


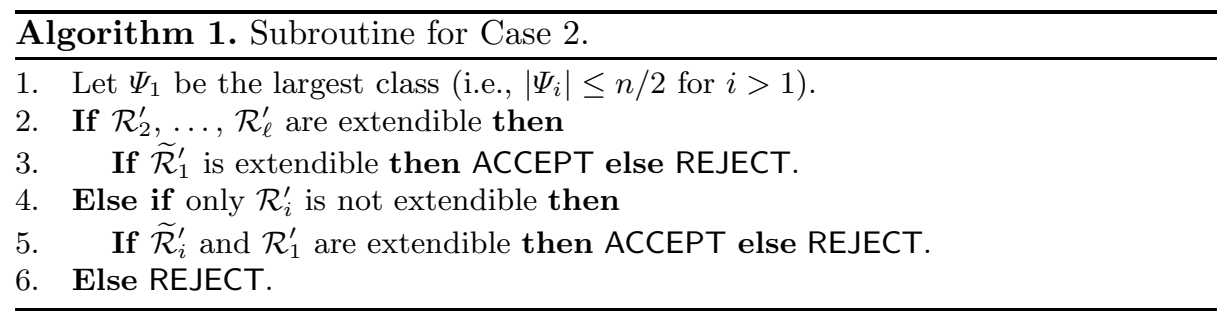

Proof (Sketch). We just put together the lemmas which are already proved; see the full version for details.

The next lemma states that the algorithm runs in polynomial time. A precise time analysis depends on algorithm used for split decomposition, and on the order in which we choose splits for recursion. We avoid this technical analysis and just note that the degree of the polynomial is reasonable small. Certainly, it would be easy to show the complexity of order $\mathcal{O}(\mathrm{nm})$.

Lemma 12. The running time of the algorithm is polynomial.

Proof (Theorem 11). The result is implied by Lemma 11 and Lemma 12.

\section{Conclusions}

The structural results described in Section [3, namely Proposition 8, are the main new tools developed in this paper. Using it, one can easily work with the structure of all representations which is a key component of the algorithm of Section 4 that solves the partial representation extension problem for circle graphs. The algorithm works with the recursive structure of all representations and matches the partial representation on it. Proposition 8 also seems to be useful in attacking the following open problems:

Question 13. What is the complexity of $\operatorname{SIM}(\mathrm{CIRCLE})$ for a fixed number $k$ of graphs? In particular, what is it for $k=2$ ?

Recall that in the bounded representation problem, we give for some chords two circular arcs and we want to construct a representation which places endpoints into these circular arcs.

Question 14. What is the complexity of the bounded representation problem for circle graphs?

\section{References}

1. Angelini, P., Battista, G.D., Frati, F., Jelínek, V., Kratochvíl, J., Patrignani, M., Rutter, I.: Testing planarity of partially embedded graphs. In: SODA 2010, pp. 202-221 (2010)

2. Balko, M., Klavík, P., Otachi, Y.: Bounded representations of interval and proper interval graphs. In: ISAAC (to appear, 2013) 
3. Bläsius, T., Rutter, I.: Simultaneous PQ-ordering with applications to constrained embedding problems. In: SODA 2013, pp. 1030-1043 (2013)

4. Bouchet, A.: Reducing prime graphs and recognizing circle graphs. Combinatorica 7(3), 243-254 (1987)

5. Bouchet, A.: Unimodularity and circle graphs. Discrete Mathematics 66(1-2), 203208 (1987)

6. Chudnovsky, M., Robertson, N., Seymour, P., Thomas, R.: The strong perfect graph theorem. Annals of Mathematics 164, 51-229 (2006)

7. Courcelle, B.: Circle graphs and monadic second-order logic. J. Applied Logic 6(3), 416-442 (2008)

8. Cunningham, W.: Decomposition of directed graphs. SIAM J. Alg. and Disc. Methods 3, 214-228 (1982)

9. Dahlhaus, E.: Parallel algorithms for hierarchical clustering and applications to split decomposition and parity graph recognition. Journal of Algorithms 36(2), 205-240 (1998)

10. de Fraysseix, H.: Local complementation and interlacement graphs. Discrete Mathematics 33(1), 29-35 (1981)

11. de Fraysseix, H., de Mendez, P.O.: On a characterization of gauss codes. Discrete \& Computational Geometry 22(2), 287-295 (1999)

12. Even, S., Itai, A.: Queues, stacks, and graphs. In: Kohavi, Z., Paz, A. (eds.) Theory of Machines and Computation, pp. 71-76 (1971)

13. Gabor, C.P., Supowit, K.J., Hsu, W.: Recognizing circle graphs in polynomial time. J. ACM 36(3), 435-473 (1989)

14. Gioan, E., Paul, C., Tedder, M., Corneil, D.: Practical and efficient circle graph recognition. Algorithmica, 1-30 (2013)

15. Jampani, K.R., Lubiw, A.: The simultaneous representation problem for chordal, comparability and permutation graphs. Journal of Graph Algortihms and Applications 16(2), 283-315 (2012)

16. Klavík, P., Kratochvíl, J., Krawczyk, T., Walczak, B.: Extending partial representations of function graphs and permutation graphs. In: Epstein, L., Ferragina, P. (eds.) ESA 2012. LNCS, vol. 7501, pp. 671-682. Springer, Heidelberg (2012)

17. Klavík, P., Kratochvíl, J., Otachi, Y., Rutter, I., Saitoh, T., Saumell, M., Vyskočil, T.: Extending partial representations of proper and unit interval graphs (in preparation, 2013)

18. Klavík, P., Kratochvíl, J., Otachi, Y., Saitoh, T.: Extending partial representations of subclasses of chordal graphs. In: Chao, K.-M., Hsu, T.-S., Lee, D.-T. (eds.) ISAAC 2012. LNCS, vol. 7676, pp. 444-454. Springer, Heidelberg (2012)

19. Klavík, P., Kratochvíl, J., Vyskočil, T.: Extending partial representations of interval graphs. In: Ogihara, M., Tarui, J. (eds.) TAMC 2011. LNCS, vol. 6648, pp. 276-285. Springer, Heidelberg (2011)

20. Kostochka, A., Kratochvíl, J.: Covering and coloring polygon-circle graphs. Discrete Mathematics 163(1-3), 299-305 (1997)

21. Naji, W.: Graphes de Cordes: Une Caracterisation et ses Applications. PhD thesis, l'Université Scientifique et Médicale de Grenoble (1985)

22. Oum, S.: Rank-width and vertex-minors. J. Comb. Theory, Ser. B 95(1), 79-100 (2005)

23. Patrignani, M.: On extending a partial straight-line drawing. In: Healy, P., Nikolov, N.S. (eds.) GD 2005. LNCS, vol. 3843, pp. 380-385. Springer, Heidelberg (2006)

24. Spinrad, J.P.: Recognition of circle graphs. J. of Algorithms 16(2), 264-282 (1994)

25. Spinrad, J.P.: Efficient Graph Representations. Field Institute Monographs (2003) 